\section{Bullous, Multifocal Fixed Drug Eruption to Pseudoephedrine: A Case Report}

\section{Abstract}

Adverse reactions to medications are extremely common and display a characteristic clinical morphology including fixed drug eruption (FDE), urticaria, Stevens-Johnson syndrome, hypersensitivity syndrome, morbilliform exanthem, pigmentary changes and so on. Fixed drug eruption is commonly seen with antimicrobials and analgesics. Here we report a case of a 45 year-old male who presented to us with multiple bullous eruptions over the trunk, upper limbs and thighs after repeated doses of pseudoephedrine.

Keywords: Fixed drug eruption, Pseudoephedrine

Received: March 08, 2016; Accepted: March 25, 2016; Published: March 29, 2016

\section{Introduction}

Fixed drug eruptions (FDE) is characterized by recurrent, welldefined and distinctive lesions in the same location each time the offending drug is ingested. Two different clinical forms have been described: A classic pigmenting form and a less commonly seen non-pigmenting form. Pseudoephedrine is known to be a major culprit of nonpigmenting FDE. To the best of our knowledge, only one case of pseudoephedrine-induced pigmenting FDE has been reported [1]. We hereby describe a case of bullous multifocal nonpigmenting FDE to pseudoephedrine. This opens a new avenue that pseudoephedrine can also develop multifocal bullous FDE which can heighten awareness among patients who constantly consume this drug and physicians who diagnose such disease.

\section{Case Report}

A 45-year-old Malay male was admitted to the National University Hospital, Singapore for a respiratory tract infection and was commenced on intravenous antibiotics. He has a history of diabetes mellitus with end- stage renal disease secondary. During this admission, he also reports a history of long-standing recurring eruptions over the same sites on his arms, chest, back and thighs. This has been occurring for the past 3 years. Of note, when these lesions recur, they would begin as fluid-filled blisters. Further history revealed the presence recurrent pseudoephedrine usage for upper respiratory tract infection with appearance of the lesions within hours to days of ingestion.

On examination, there were multiple well-defined oval erythematous patches over both palms. A flaccid blister was

\section{Adeline Mei-Yen Yong and Sam Shiyao Yang}

\author{
Department of Dermatology, Nationa \\ University Health System, Singapore
}

\section{Corresponding author: \\ Adeline Mei-Yen Yong \\ Adeline_meiyen@hotmail.com \\ Department of Dermatology, National University Health System, 5 Lower Kent Ridge Rd, Singapore.}

Tel: +6584489319

Citation: Yong AM, Yang SS. Bullous, Multifocal Fixed Drug Eruption to Pseudoephedrine: A Case Report. Ann Clin Lab Res. 2016, 4:1.

noted over his left palm. There were no erosions over the lips or involvement of the genitals. There were also round pigmented patches over his forearms, thighs and anterior trunk, which were sites where the same eruption occurred previously, representing areas of post inflammatory hyperpigmentation. Skin prick, intradermal and patch tests were negative for common culprits including Augmentin, Penicillin, Piperacillin/Tazobactam and Ceftriaxone (Figures 1 - 5).

The diagnosis of multifocal pseudoephedrine-induced bullous FDE was based on clinical grounds along with his past history of similar recurring lesions on the same body areas over the past 3 years whenever he took pseudoephedrine for episodes of upper respiratory infection. The lesions resolved shortly after cessation of the inciting agent and he was advised to avoid the drug in the future.

\section{Discussion}

FDE is characterized by pruritic, well-circumscribed, erythematous patches at the same site with re-exposure to an offending drug. Two different clinical forms have been described: the common classic pigmenting form and the rare nonpigmenting form. Pseudoephedrine hydrochloride is the drug most commonly associated with non-pigmenting FDE and the bullous form was first described in 1 of the 3 cases reported by Shelley and Shelley [2]. This case of FDE is unusual in that it the FDE are multifocal, 


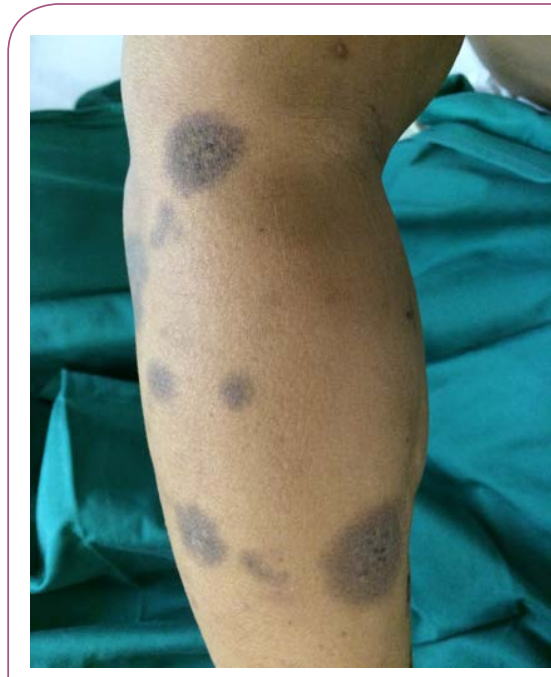

Figure 1 Multiple well-defined annular erythematous plaques over left forearm.

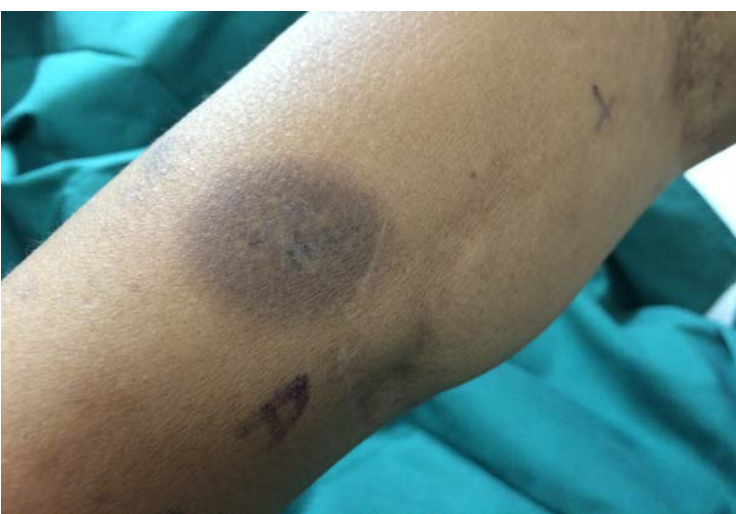

Figure 2 Close-up of plaque over left forearm.

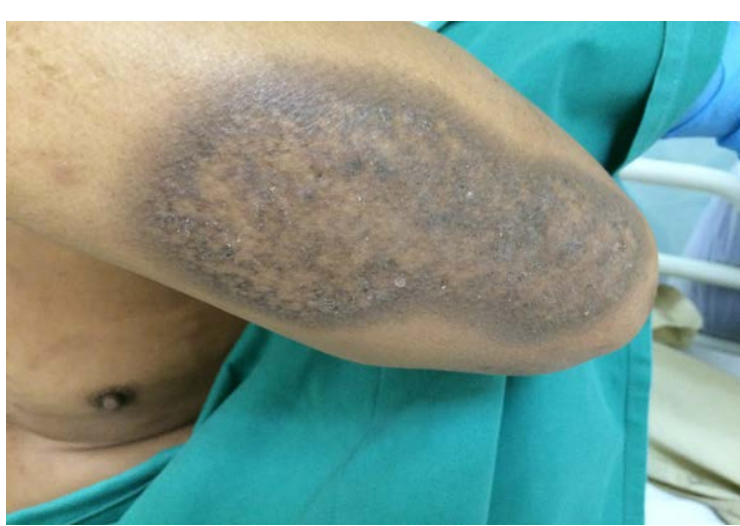

Figure 3 Large, annular hyperpigmented plaque with areas of central variegation over lateral aspect of right arm.
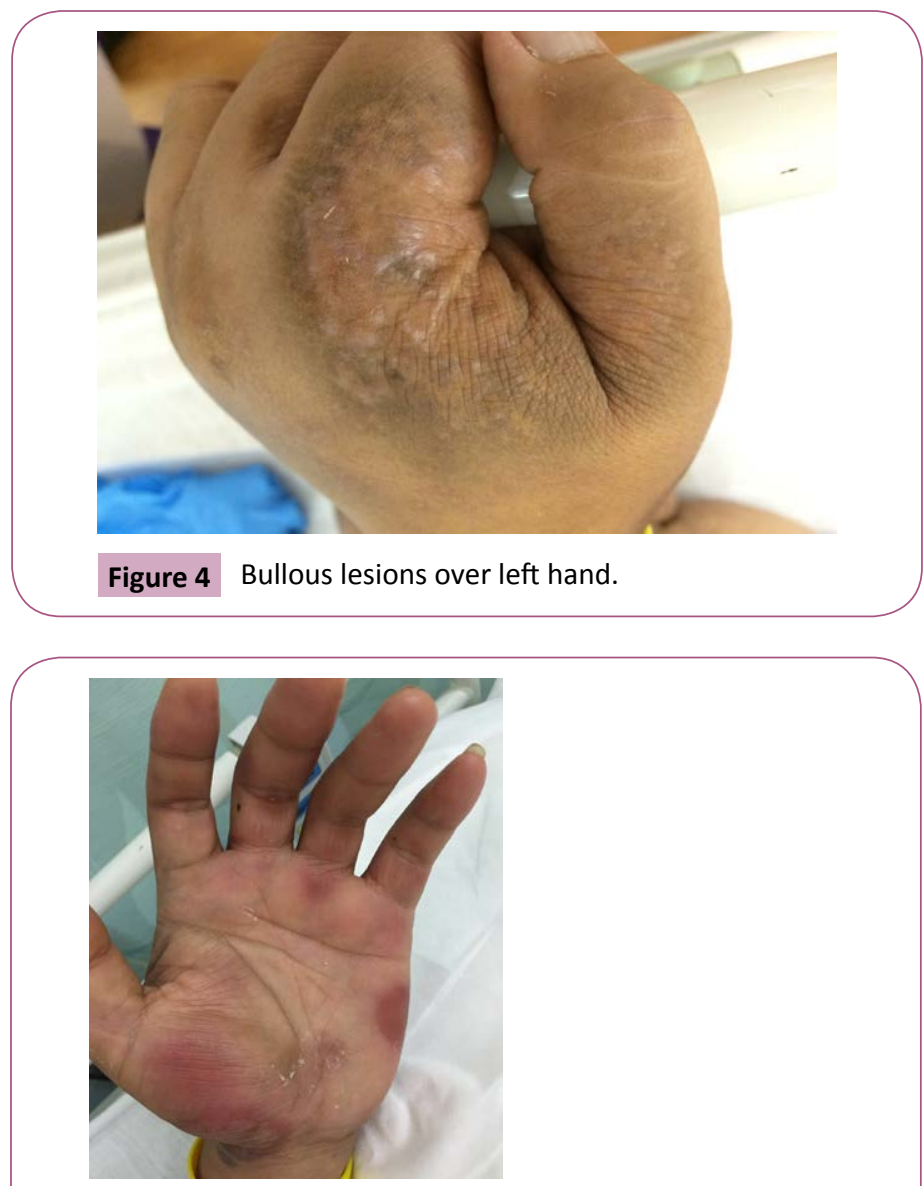

Figure 5 Multifocal erythematous patches over left palm.

and was associated with bulla formation. To our knowledge, among the cases of pseudoephedrine- induced FDE reported in the literature, ours is the third bullous form described $[3,4]$.

In addition, the half-life elimination of pseudoephedrine varies by urine $\mathrm{pH}$ and flow rate as $43-96 \%$ is excreted in the urine as unchanged drug form and $1-6 \%$ excreted as active norpseudoephedrine [5]. Alkaline urine decreases renal elimination of pseudoephedrine. It is hence likely that the history of end-stage renal disease of this patient aggravated the side effects of pseudoephedrine.

The FDE is one of the most common types of drug eruption; of which incidence of FDE was reported to constitute less than $1 \%$ of all dermatologic problems [5]. However, there are only few case reports on multifocal FDE. This case illustrates a clinically important and rare cutaneous drug reaction of pseudoephedrine; a commonly prescribed decongestant. Hence, it should be kept in mind that use of pseudoephedrine can result in development of multifocal FDE and should be prescribed carefully. 


\section{References}

1 Ozkaya E, Elinc-Aslan MS (2011) Pseudoephedrine may cause "pigmenting" fixed drug eruption. Dermatitis: contact, atopic, occupational, Drug 22: E7-E9.

2 Shelley WB, Shelley ED (1987) Nonpigmenting fixed drug eruption as a distinctive reaction pattern: examples caused by sensitivity to pseudoephedrine hydrochloride and tetrahydrozoline. Journal of the American Academy of Dermatology 17: 403-407.

3 Bellini V, Bianchi L, Hansel K, Finocchi R, Stingeni L (2016)
Bullous nonpigmenting multifocal fixed drug eruption due to pseudoephedrine in a combination drug: clinical and diagnostic observations. The journal of allergy and clinical immunology, In practice.

4 Anadkat MJ, Cowen EW, Mercurio MG, Scott GA (2003) Disseminated bullous fixed drug eruption caused by sympathomimetic medications: ma huang, phenylpropanolamine, and pseudoephedrine. Journal of the American Academy of Dermatology 49: E6-E9.

5 Kanfer I, Dowse R, Vuma V (1993) Pharmacokinetics of oral decongestants. Pharmacotherapy 13: 116S-128S; discussion 143S-146S. 